\title{
ON THE DISTRIBUTION OF THE SURPLUS PRIOR TO RUIN IN A DISCRETE SEMI-MARKOV RISK MODEL
}

\author{
BY \\ J.M. ReINHARD* AND M. SNOUSSi*• \\ * Université Libre de Bruxelles \\ - Secura Belgian Re, Belgique
}

\begin{abstract}
In this paper we extend the work of Reinhard and Snoussi (2000) by developing a recursive system for finding the distribution of the surplus prior to ruin in a discrete semi-Markov risk model.
\end{abstract}

KEYWORDS

Probability of ruin; surplus prior to ruin; recursive calculation; stable algorithm.

\section{INTRODUCTION}

In classical risk theory, various quantities for a classical surplus process have been treated in the actuarial literature in various kinds of risk models. These quantities are the probability of ultimate ruin, the distribution of the severity of ruin, the distribution of the surplus immediately prior to ruin and the joint distribution of the surplus immediately prior to ruin and the severity of ruin. The study of these quantities has been extensively treated in recent years, some key references being Bowers et al. (1987), Gerber (1979, 1988), Gerber et al. (1997, 1998), Dickson (1992), Dickson et al. (1992), Dufresne and Gerber (1988), Klugman et al. (1998), Reinhard (1984), Snoussi (1998).

In this paper we are interested in the distribution of the surplus prior to ruin in a discrete semi Markov risk model. The classical results concerning this distribution for the continuous time risk model were first discussed in Dufresne (1989). This work was extended by several authors such as Gerber and Dufresne (1988), Dickson (1992), Willmot and Lin (1998) and Gerber and Shiu (1998).

Dickson (1992) and Dickson et al. (1995) study the distribution of the surplus prior to ruin in a fully discrete claims model where time, claim amounts, premium and initial surplus are assumed to be integer valued. Our purpose in 
this paper is to extend this work to a more general discrete model where we assume that the claims are influenced by a Markov chain (see Reinhard and Snoussi (2000)). Assumptions of this kind have been discovered in a Markov modulated model where the intensity and the premium can fluctuate according to a Markovian environment. References we may cite on this topic are Asmussen (1989), Grandell (1991), Janssen and Reinhard (1985), Reinhard (1984) and Snoussi (1998). This type of modelling motivates the present work.

\section{THE DISCRETE SEMI-MARKOV RISK MODEL}

In this section we are interested in a discrete time semi-Markov risk model introduced by Reinhard and Snoussi (2000), and defined as follows: Denote by $\left(J_{n}, n \in \mathbb{N}\right)$ a homogeneous, irreducible and aperiodic Markov chain with finite state space $M=\{1, \ldots, m\}$ (with $1 \leq m<\infty$ ) which influences the distribution of the claims in each period. The variable $J_{n}$ can be interpreted as the type of economic environment for period $n$. Asmussen (1989) discusses car insurance where the states of $\left(J_{n}, n \in \mathbb{N}\right)$ describe weather conditions. Denote by $P=\left(p_{i j}\right)_{i, j \in\{1, \ldots, m\}}$ the transition probability matrix of $\left(J_{n}\right)$ :

$$
p_{i j}=\mathbb{P}\left(J_{n}=j \mid J_{n-1}=i, J_{k} \quad k \leq n-1\right),
$$

and by $\pi=\left(\pi_{1}, \ldots, \pi_{m}\right)$ its unique stationary probability distribution. The insurer's surplus at the end of the $t$-th period $(t=1,2, \ldots), Z_{t}$, satisfies the following relation:

$$
Z_{t}=Z_{t-1}+c-X_{t}(t=1,2, \ldots),
$$

where $X_{t}$ denotes the total amount of claims for the $t$-th period $(t=1,2, \ldots)$ and $c$ the total premium for each period. We suppose that the insurer's initial surplus $Z_{0} \in \mathbb{N}=\{0,1,2, \ldots\}, c \in \mathbb{N}$ and that the $X_{t}^{\prime}$ s are positive and integervalued random variables, conditionally independent given the Markov chain $\left(J_{n}, n \in \mathbb{N}\right)$. Moreover we suppose that $\left(J_{t}, X_{t}\right)$ depends on $\left\{J_{k}, X_{k} ; k \leq t-1\right\}$ only through $J_{t-1}$. Let

$$
g_{i j}(k)=\mathbb{P}\left(X_{t}=k, J_{t}=j \mid J_{t-1}=i, J_{k}, X_{k} \quad k \leq t-1\right),
$$

and

$$
\begin{aligned}
& g_{i}(k)=\sum_{j=1}^{m} g_{i j}(k), \\
& G_{i}(k)=\sum_{j=1}^{m} G_{i j}(k)=\sum_{j=1}^{m} \sum_{u=0}^{k} g_{i j}(u),
\end{aligned}
$$

for $i=1, \ldots, m, k \geq 0$. We suppose that there exists at least one pair $\{i, j\}$ such that $p_{i j}>0$ and the distribution of $X_{t}$ given $J_{t-1}=i$ and $J_{t}=j$ is not degenerate. 
In this case the process $\left\{\left(J_{t}, Z_{t}-Z_{0}\right), t \in \mathbb{N}\right\}$ is not degenerate (see Newbould (1973)).

Assume further that for all $i$ and $j$

$$
\gamma_{i j}=E\left(X_{t} 1_{\left[J_{t}=j\right]} \mid J_{t-1}=i\right)=\sum_{k=1}^{\infty} k g_{i j}(k)<\infty,
$$

and define

$$
\gamma^{*}=\sum_{i \in M} \pi_{i} \sum_{j \in M} \gamma_{i j}
$$

If the distribution of $J_{0}$ is $\pi$, we have

$$
\gamma^{*}=E\left(X_{t}\right) \quad(\forall t=1,2, \ldots) .
$$

It is well known that $\pi_{j}^{-1}\left(c-\gamma^{*}\right)$ is the expected increase of the surplus process $\left\{Z_{t}, t=0,1,2, \ldots\right\}$ between two successive visits of the Markov cháin $\left(j_{n}, n \in \mathbb{N}\right)$ to state $j$ (see for example theorem 2 in Reinhard, 1984).

Let $T$ be the time of ruin:

$$
T=\inf \left\{t \in \mathbb{N} \mid Z_{t}<0\right\} .
$$

We are interested in the ruin probabilities

$$
\psi_{i}(u)=\mathbb{P}\left(T<\infty \mid Z_{0}=u, J_{0}=i\right) \quad(u \in \mathbb{N} ; i=1, \ldots, m) .
$$

$\psi_{i}(u)$ represents the ultimate ruin probability given that the initial surplus is $u$ and that the Markov chain $\left(J_{n}\right)$ starts in state $i$. Let $\Phi_{i}(u)=1-\psi_{i}(u)$ be the corresponding survival probability. It follows easily from Janssen (1970, theorem 3A) or Newbould (1973, theorem 1) that

- If $\gamma^{*}<c$ then $\lim _{n \rightarrow \infty} Z_{n}=\infty$ a.s. and $\psi_{i}(\infty)=\lim _{u \rightarrow \infty} \psi_{i}(u)=0, \forall i \in\{1, \ldots, m\}$,

- If $\gamma^{*}>c$ then $\lim _{n \rightarrow \infty} Z_{n}=-\infty$ a.s. and $\psi_{i}(u)=1, \forall i \in\{1, \ldots, m\}$ and $\forall u \geq 0$,

- If $\gamma^{*}=c$ then $\varlimsup_{n \rightarrow \infty} Z_{n}=\infty$ a.s., $\underline{\lim }_{n \rightarrow \infty} Z_{n}=-\infty$ a.s. and $\psi_{i}(u)=1, \forall i \in$ $\{1, \ldots, m\}$ and $\forall u \geq 0$.

We suppose therefore that $\gamma^{*}<c$.

We will also consider the quantity $Z_{T-1}$ which is the surplus prior to ruin.

We define therefore

$$
\mathcal{F}_{i}(u, x)=\mathbb{P}\left(T<\infty, Z_{T-1}<x \mid Z_{0}=u, J_{0}=i\right) .
$$

If the values of $\mathcal{F}_{i}(u, x)$ are known, we can use them to calculate the probabilities of ruin $\Psi_{i}(u)$ as

$$
\Psi_{i}(u)=\lim _{x \rightarrow \infty} \mathscr{F}_{i}(u, x) \quad(u \in \mathbb{N}, i \in M)
$$


We shall use the following notation throughout this paper. Let $A=\left(a_{i j}\right)_{i, j \in\{1, \ldots, m\}}$ be a matrix in $\mathbb{R}^{m \times m}$, then we denote by $\bar{A} \cdot 1$ the vector $\left(a_{21}, \ldots, a_{m !}\right)^{t}$ in $\mathbb{R}^{m-1}$, $\bar{A}_{\cdot 1}$ will denote the vector $\left(a_{12}, \ldots, a_{1 m}\right)^{t}$ in $\mathbb{R}^{m-1}$. The matrix $\left(a_{i j}\right)_{i, j \in\{2, \ldots, m\}}$ in $\mathbb{R}^{(m-1) \times(m-1)}$ is denoted by $A^{(1)}$. Finally, we denote by $\mathbb{N}$ the set of the non-negative integers, and by $\mathbb{N}_{0}$ the set of the non-null integers.

\section{ReCURSive Formula in A PARTICULAR CASE WITH C $=1$}

In this section we assume that the annual premium $c=1$ and the annual result $c-X_{t}(t=1,2, \ldots)$ may be positive only in periods starting in state 1 . Therefore, we will assume in the sequel of this paper the following condition:

$$
\left\{\begin{array}{l}
g_{i j}(0)=0 \\
\sum_{j \in M} g_{1 j}(0)>0 .
\end{array}(\forall j \in M, i \neq 1),\right.
$$

Note that the assumption $c=1$ could be motivated with the argument that one is free to choose the unit time interval. The restriction on the total claim sizes (12) is essential to derive an explicit formula for $\mathcal{F}_{i}(0, x)$. One possible application could be an insurance contract where, when the insured is in state 1 (e.g. healthy) he pays a premium $c$, and when the insured is in state 2 (e.g. sickness), the payment of the premium is interrupted and the insured receives the contract benefits.

In Subsections 3.1 and 3.2, we give an explicit formula for $\mathcal{F}_{i}(0, x)$ (with $\left.x \in \mathbb{N}_{0}\right)$ in the $m$ states model and we show how to calculate $\mathcal{F}_{i}(u, x)$ for $u \in \mathbb{N}_{0}$, by a stable recursive system. In Subsection 3.3, we give a recursive stable algorithm to compute the joint distribution of surplus prior to ruin and the severity of ruin. Finally, in Subsection 3.4, some numerical examples are presented to illustrate our results in the two-state model (the case $m=2$, i.e. the environment process has only two states).

\subsection{Model with $m$ states}

By conditioning with respect to the first period's result, it is easily seen that the probability that ruin occurs from initial surplus $(u \in \mathbb{N})$ in state $i$ and that the surplus prior to ruin is less than $x$ (with $x \in \mathbb{N}_{0}$ ), satisfies the following equations:

$$
\left\{\begin{array}{l}
\mathcal{F}_{i}(u, x)=\sum_{j=1}^{m} \sum_{k=0}^{u+1} g_{i j}(k) \mathcal{F}_{j}(u+1-k, x)+\sum_{k=u+2}^{\infty} g_{i}(k), \text { if } u<x \\
\mathcal{F}_{i}(u, x)=\sum_{j=1}^{m} \sum_{k=0}^{u+1} g_{i j}(k) \mathcal{F}_{j}(u+1-k, x), \text { if } u \geq x,(i=1, \ldots, m) .
\end{array}\right.
$$


In order to solve the above system under condition (12), we introduce the following probabilities:

$$
\mathbb{K}_{i}(u, v)=\mathbb{P}\left(Z_{\tau}=v \mid Z_{0}=u, J_{0}=i\right) \quad(i \in\{2, \ldots, m\}),
$$

where

$$
\tau=\inf \left\{n \in \mathbb{N} \mid J_{n}=1\right\}
$$

As pointed out by Reinhard and Snoussi $(2000), \mathbb{K}_{i}(u, v)$ depends only on the difference $u-v$. Therefore, we define for $d \in \mathbb{N}: \mathbb{K}_{i}(d)=\mathbb{K}_{i}(u, u-d)$.

Let

$$
\begin{array}{ll}
\overline{\mathbb{K}}(d)=\left(\mathbb{K}_{2}(d), \ldots, \mathbb{K}_{m}(d)\right)^{t} & \in \mathbb{R}^{m-1}, \\
g(k)=\left(g_{i j}(k)\right)_{i, j \in\{1, \ldots, m\}} & \in \mathbb{R}^{m \times m} .
\end{array}
$$

It can be shown (see Reinhard and Snoussi (2000), equation (29)) that $\mathbb{K}_{i}(d)$, $d \in \mathbb{N}, i \in\{2, \ldots, m\}\}$ may be calculated through the following stable recursive system:

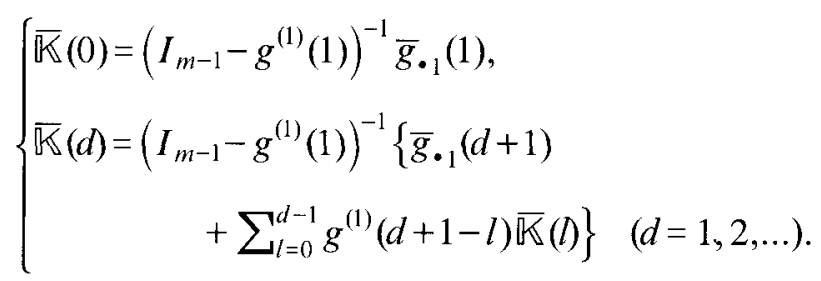

Note that $\left(J_{n}, n \in N\right)$ is ergodic. Therefore $I_{m-1}-g^{(1)}(1)$ is invertible, where $I_{m-1}$ is the unit matrix in $\mathbb{R}^{(m-1) \times(m-1)}$.

Next, we define for $u=0,1,2, \ldots$ and $i \in\{2, \ldots, m\}$

$$
\mathcal{F}_{i}^{*}(u, x)=\mathbb{P}\left(T \leq \tau, Z_{T-1}<x \mid Z_{0}=u, J_{0}=i\right) \quad\left(\forall x \in \mathbb{N}_{0}\right),
$$

to be the probability that, starting with surplus $u$ in state $i, i \in\{2, \ldots, m\}$, ruin occurs before or at the first visit to state 1 and that the surplus prior to ruin is less than $x$, where $x>0$. Hence, by the law of total probability, we get for $u \geq 0$ and $x \in \mathbb{N}_{0}$

$$
\begin{cases}\mathcal{F}_{i}^{*}(u, x)=\sum_{j=2}^{m} \sum_{l=1}^{u+1} g_{i j}(l) \mathcal{F}_{j}^{*}(u+1-l, x)+\sum_{l=u+2}^{\infty} g_{i}(l), & \text { if } u<x, \\ \mathcal{F}_{i}^{*}(u, x)=\sum_{j=2}^{m} \sum_{l=1}^{u+1} g_{i j}(l) \mathcal{F}_{j}^{*}(u+1-l, x), & \text { if } u \geq x .\end{cases}
$$


We define

$$
\begin{array}{ll}
\overline{\mathcal{F}}^{*}(u, x)=\left(\mathcal{F}_{2}^{*}(u, x), \ldots, \mathcal{F}_{m}^{*}(u, x)\right)^{t} & \in \mathbb{R}^{m-1}, \\
\bar{g}(u)=\left(g_{2}(u), \ldots, g_{m}(u)\right)^{t} & \in \mathbb{R}^{m-1} .
\end{array}
$$

Thus, we have that $\left\{\mathcal{F}_{i}^{*}(u, x), u \in \mathbb{N}, x \in \mathbb{N}_{0}, i \in\{2, \ldots, m\}\right\}$ may be calculated by the following stable recursive system

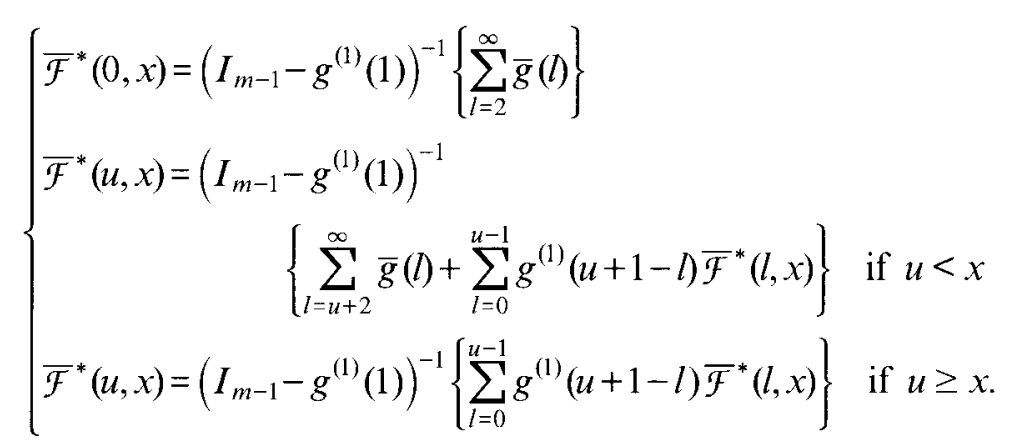

We have for $u \geq 0$ and $x \in \mathbb{N}_{0}$ :

$$
\mathcal{F}_{i}(u, x)=\sum_{v=0}^{u} \mathbb{K}_{i}(u-v) \mathcal{F}_{1}(v, x)+\mathcal{F}_{i}^{*}(u, x)
$$

If starting with surplus $u$ in a state $i(i \in\{2, \ldots, m\})$, ruin occurs with a surplus prior to ruin is less than $x$, then

- either, ruin occurs before or at the first visit to state 1 and the surplus before ruin is less than $x$;

- or, the first visit to state 1 occurs with a positive surplus $v$ and thereafter ruin occurs from this new surplus with surplus before ruin is less than $x$.

The probability of the first event is $\mathcal{F}_{i}^{*}(u, x)$ and the probability of the second event is the first term in the right-hand side of (20).

Now it remains to calculate $\mathcal{F}_{1}(\cdot, x)$. Introducing (20) into (13) with $i=1$, we obtain: for $u<x$

$$
\mathcal{F}_{1}(u, x)=\sum_{l=0}^{u+1} f(l) \mathcal{F}_{1}(u+1-l, x)+\sum_{j=2}^{m} \sum_{l=0}^{u+1} g_{1 j}(l) \mathcal{F}_{j}^{*}(u+1-l, x)+\sum_{k=u+2}^{\infty} g_{1}(k),
$$

and, for $u \geq x$ :

$$
\mathcal{F}_{1}(u, x)=\sum_{l=0}^{u+1} f(l) \mathcal{F}_{1}(u+1-l, x)+\sum_{j=2}^{m} \sum_{l=0}^{u+1} g_{1 j}(l) \mathcal{F}_{j}^{*}(u+1-l, x),
$$


where

$$
f(l)=g_{11}(l)+\sum_{j=2}^{m} \sum_{v=0}^{l} g_{1 j}(v) \mathbb{K}_{j}(l-v)
$$

Rearranging (21) and (22) yields for $u \in \mathbb{N}_{0}$, if $u \leq x$ :

$$
\begin{gathered}
\mathcal{F}_{1}(u, x)=\frac{1}{f(0)}\left[\mathcal{F}_{1}(u-1, x)-\sum_{l=0}^{u-1} f(u-l) \mathcal{F}_{1}(l, x)\right. \\
\left.-\sum_{j=2}^{m} \sum_{l=0}^{u} g_{1 j}(l) \mathcal{F}_{j}^{*}(u-l, x)-1+G_{1}(u)\right]
\end{gathered}
$$

and, if $u>x$

$$
\begin{gathered}
\mathcal{F}_{l}(u+1, x)=\frac{1}{f(0)}\left[\mathcal{F}_{1}(u, x)-\sum_{l=0}^{u} f(u-l+1) \mathcal{F}_{1}(l, x)\right. \\
\left.-\sum_{j=2}^{m} \sum_{l=0}^{u+1} g_{l j}(l) \mathcal{F}_{j}^{*}(u-l+1, x)\right] .
\end{gathered}
$$

Formulae (24) and (25) can be used to calculate $\mathcal{F}_{1}(u, x)$ recursively provided that the value of $\mathcal{F}_{1}(0, x)$ is known. Using an approach similar to that used by Reinhard and Snoussi (1998), for a fixed value $x$, we define

$$
\begin{aligned}
& \left\{\begin{array}{l}
d(0)=\mathcal{F}_{1}(0, x) \\
d(u)=\mathcal{F}_{1}(u, x)-\mathcal{F}_{1}(u-1, x),
\end{array}\right. \\
& \left\{\begin{array}{l}
f_{i}^{*}(0)=\mathcal{F}_{i}^{*}(0, x) . \\
f_{i}^{*}(u)=\mathcal{F}_{i}^{*}(u, x)-\mathcal{F}_{i}^{*}(u-1, x)
\end{array}(u=1,2, \ldots, i \in\{2, \ldots, m\}) .\right.
\end{aligned}
$$

It is straightforward to show that:

$$
\begin{aligned}
d(0)= & f(0) d(1)+(f(0)+f(1)) d(0)+\sum_{j=2}^{m} g_{1 j}(0) f_{j}^{*}(1) \\
& +\sum_{j=2}^{m}\left(g_{1 j}(0)+g_{1 j}(1)\right) f_{j}^{*}(0)+\sum_{l=2}^{\infty} g_{1}(l) .
\end{aligned}
$$

Then, for $0<u<x$,

$$
d(u)=\sum_{l=0}^{u+l} f(l) d(u+1-l)+\sum_{j=2}^{m} \sum_{l=0}^{u+1} g_{1 j}(l) f_{j}^{*}(u+1-l)-g_{1}(u+1) .
$$


For $u=x$

$$
d(u)=\sum_{l=0}^{u+1} f(l) d(u+1-l)+\sum_{j=2}^{m} \sum_{l=0}^{u+1} g_{1 j}(l) f_{j}^{*}(u+1-l)-\sum_{l=u+1}^{\infty} g_{1}(l) .
$$

For $u>x$

$$
d(u)=\sum_{l=0}^{u+1} f(l) d(u+1-l)+\sum_{j=2}^{m} \sum_{l=0}^{u+1} g_{1 j}(l) f_{j}^{*}(u+1-l) .
$$

We also define these generating functions

$$
\begin{aligned}
& \delta(s)=\sum_{x=0}^{\infty} s^{x} d(x), \delta_{i}^{*}(s)=\sum_{x=0}^{\infty} s^{x} f_{i}^{*}(x), \varphi(s)=\sum_{x=0}^{\infty} s^{x} f(x) \text { and } \\
& w_{i j}(s)=\sum_{x=0}^{\infty} s^{x} g_{i j}(x) .
\end{aligned}
$$

So we can write

$$
\begin{aligned}
\delta(s) & =d(0)+\sum_{r=1}^{\infty} s^{r} d(r) \\
= & \sum_{r=1}^{\infty} s^{r}\left[\sum_{l=0}^{r+1}\left[f(l) d(r+1-l)+\sum_{j=2}^{m} g_{1 j}(l) f_{j}^{*}(r+1-l)\right]\right] \\
& -\sum_{r=0}^{x-1} s^{r} g_{1}(r+1)-s^{x} \sum_{l=x+1}^{\infty} g_{1}(l)+f(0) d(1)+(f(0)+f(1)) d(0) \\
& +\sum_{j=2}^{m} g_{1 j}(0) f_{j}^{*}(1)+\sum_{j=2}^{m}\left(g_{1 j}(0)+g_{1 j}(1)\right) f_{j}^{*}(0)+\sum_{l=1}^{\infty} g_{1}(l) .
\end{aligned}
$$

Then, by interchanging the order of summation, it follows

$$
\begin{gathered}
\delta(s)=s^{-1} \varphi(s) \delta(s)+s^{-1} \sum_{j=2}^{m} w_{1 j}(s) \delta_{j}^{*}(s)+\left(1-s^{-1}\right)\left[f(0) d(0)+\sum_{j=2}^{m} g_{1 j}(0) f_{j}^{*}(0)\right] \\
-\sum_{r=0}^{x-1} s^{r} g_{1}(r+1)-\sum_{l=x+1}^{\infty} s^{x} g_{1}(l)+\sum_{l=1}^{\infty} g_{1}(l) .
\end{gathered}
$$

Finally, we obtain

$$
\begin{gathered}
\delta(s)=\frac{\left(1-s^{-1}\right)\left[f(0) d(0)+\sum_{j=2}^{m} g_{1 j}(0) f_{j}^{*}(0)\right]+s^{-1} \sum_{j=2}^{m} w_{1 j}(s) \delta_{j}^{*}(s)}{\left(1-s^{-1} \varphi(s)\right)} \\
-\frac{\sum_{r=0}^{x-1} s^{r} g_{1}(r+1)+\sum_{l=x+1}^{\infty} s^{x} g_{1}(l)-\sum_{l=1}^{\infty} g_{1}(l)}{\left(1-s^{-1} \varphi(s)\right)} .
\end{gathered}
$$


Since $\delta(1)=\mathcal{F}_{1}(\infty, x)=0, \varphi(1)=1$, for $i \in\{2, \ldots, m\}, w_{1 i}(1)=p_{1 i}$ and $\delta_{i}^{*}(1)=$ $\mathcal{F}_{i}^{*}(\infty, x)=0$, both the numerator and denominator go to zero when $s \rightarrow 1$. Using $\varphi^{\prime}(1) \neq 1$ [see Reinhard and Snoussi (1998)] and applying l'Hospital's rule when $s \rightarrow 1$, we get:

$$
\begin{aligned}
0=f(0) d(0) & +\sum_{j=2}^{m} g_{1 j}(0) f_{j}^{*}(0)+\sum_{j=2}^{m} p_{1 j} \sum_{x=0} x f_{j}^{*}(x) \\
& -\sum_{r=0}^{x-1} r g_{1}(r+1)-x \sum_{r=x+1}^{\infty} g_{1}(r) .
\end{aligned}
$$

Then, we have

$$
\begin{gathered}
\mathcal{F}_{1}(0, x)=\frac{1}{f(0)}\left[\sum_{r=0}^{x-1} r g_{1}(r+1)+x \sum_{r=x+1}^{\infty} g_{1}(r)-\left(\bar{g}_{1} \cdot(0)\right)^{t}\right. \\
\left.\left(I_{m-1}-g^{(1)}(1)\right)^{-1}\left\{\sum_{l=2}^{\infty} \bar{g}(l)\right\}-\sum_{j=2}^{m} p_{1 j} \sum_{x=0}^{\infty} x f_{j}^{*}(x)\right] .
\end{gathered}
$$

Formula (28) allows us to calculate $\mathcal{F}_{1}(0, x)$ provided that the value of $\sum_{x=0} x f_{j}^{*}(x)$ for $j \in\{2, \ldots, m\}$ and $\sum_{r=0}^{x-1} r g_{1}(r+1)+x \sum_{r=x+1}^{\infty} g_{1}(r)$ are known. It is easy to obtain,

$$
\begin{aligned}
\sum_{r=0}^{x-1} r g_{1}(r+1)+x \sum_{r=x+1}^{\infty} g_{1}(r) & =\sum_{r=0}^{x-1} r g_{1}(r+1)+x\left(1-\sum_{r=0}^{x} g_{1}(r)\right) \\
& =-\sum_{l=1}^{x-1} \sum_{r=1}^{l} g_{1}(r)+\sum_{r=1}^{x}\left(1-g_{1}(0)\right) \\
& =\sum_{r=0}^{x-1}\left(1-G_{1}(r+1)\right) .
\end{aligned}
$$

We note that

$$
\sum_{u=0}^{\infty} u f_{i}^{*}(u)=-\sum_{u=0}^{\infty} \mathcal{F}_{i}^{*}(u, x)
$$

Therefore, if we let $\bar{\sigma}(x)=\left(\sum_{u=0}^{\infty} \mathcal{F}_{2}^{*}(u, x), \ldots, \sum_{u=0}^{\infty} \mathcal{F}_{m}^{*}(u, x)\right)^{t}$ and $\bar{e}=(1, \ldots, 1)^{t}$, $\in \mathbb{R}^{m-1}$ we can write

$$
\begin{aligned}
\bar{\sigma}(x) & =\left(I_{m-1}-g^{(1)}(1)\right)^{-1}\left(\sum_{u=0}^{x-1} \sum_{l=u+2}^{\infty} \bar{g}(l)+\sum_{u=1}^{\infty} \sum_{l=0}^{u-1} g^{(1)}(u+1-l) \overline{\mathcal{F}}^{*}(l, x)\right) \\
& =\left(I_{m-1}-g^{(1)}(1)\right)^{-1}\left(\sum_{u=0}^{x-1}(\bar{e}-\bar{G}(u+1))+\sum_{u=2}^{\infty} g^{(1)}(u) \bar{\sigma}(x)\right),
\end{aligned}
$$

where $\bar{G}(k)=\sum_{u=0}^{k} \bar{g}(u)$. 
Hence,

$$
\bar{\sigma}(x)=\left(I_{m-1}-P^{(1)}\right)^{-1} \sum_{u=0}^{x-1}(\bar{e}-\bar{G}(u+1)) .
$$

Consequently, the value of $\mathcal{F}_{1}(0, x)$, for $x \in \mathbb{N}_{0}$, is explicitly given by

$$
\begin{gathered}
\mathcal{F}_{1}(0, x)=\frac{1}{f(0)}\left[\sum_{r=0}^{x-1}\left(1-G_{1}(r+1)\right)-\left(\bar{g}_{1} \cdot(0)\right)^{t}\left(I_{m-1}-g^{(1)}(1)\right)^{-1}\right. \\
\left.\left\{\sum_{l=2}^{\infty} \bar{g}(l)\right\}+\sum_{u=0}^{x-1}\left(\bar{P}_{1} \cdot\right)^{t}\left(I_{m-1}-P^{(1)}\right)^{-1}(\bar{e}-\bar{G}(u+1))\right] .
\end{gathered}
$$

Although we experienced that this algorithm provides very good approximations for moderate values of $u$, unfortunately we have some doubts about stability (see Panjer and Wang (1993)) of the recursive scheme based on formulae (24) and (25). Therefore we may not exclude that in some particular cases, the rounding errors could blow up, producing meaningless results. For example the algorithm could produce computed values of $\mathcal{F}_{1}(u, x)$ (for $u \in \mathbb{N}$ and $\left.x \in \mathbb{N}_{0}\right)$ outside the interval $[0,1]$. An alternative stable recursive method to calculate $\mathcal{F}_{1}(u, x)$ is proposed in the following subsection.

\subsection{Stable Algorithm}

Starting from

$$
\begin{gathered}
\mathcal{F}_{1}(0, x)=\frac{1}{f(0)}\left[\sum_{r=0}^{x-1}\left(1-G_{1}(r+1)\right)-\left(\bar{g}_{1} \cdot(0)\right)^{t}\left(I_{m-1}-g^{(1)}(1)\right)^{-1}\right. \\
\left.\left\{\sum_{l=2}^{\infty} \bar{g}(l)\right\}+\sum_{u=0}^{x-1}\left(\bar{P}_{1} \bullet\right)^{t}\left(I_{m-1}-P^{(1)}\right)^{-1}(\bar{e}-\bar{G}(u+1))\right] .
\end{gathered}
$$

We can calculate $\mathcal{F}_{1}(u, x)$ for $x \in \mathbb{N}_{0}$ from the following formula:

For $u<x$

$$
\mathcal{F}_{1}(u, x)=\sum_{j=1}^{m} \sum_{y=1}^{u} \psi_{1 j}(0, y) \mathcal{F}_{j}(u-y, x)+\sum_{v=0}^{x-u-1} \sum_{y=u+1}^{\infty} f_{1}(0, v, y),
$$

where in the right member of the last equality we define

$$
\begin{gathered}
f_{i}(u, x, y)=\mathbb{P}\left(T<\infty, Z_{T}=-y, Z_{T-1}=x \mid Z_{0}=u, J_{0}=i\right) \\
(\forall u \geq 0, i \in\{1, \ldots, m\}),
\end{gathered}
$$

to be the joint density of the surplus prior to ruin and the severity of ruin. 
For $u \geq x$, we have

$$
\mathcal{F}_{1}(u, x)=\sum_{j=1}^{m} \sum_{y=1}^{u} \psi_{1 j}(0, y) \mathcal{F}_{j}(u-y, x) .
$$

Formula (35) follows by considering the first time on which the surplus falls below its initial level. The first term of (33) follows from the similar argument. The second term in (33) comes from considering the situation when ruin occurs on the first time that the surplus falls below its initial level.

Note that, $\psi_{1 j}(0, y)$ for $y \geq 1$ and $j \in\{1, \ldots, m\}$, are calculated explicitly from Reinhard and Snoussi (1998), that is:

$$
\begin{gathered}
\psi_{11}(0, y)=\frac{1}{f(0)}\left[p_{11}-G_{11}(y)-\left(\bar{g}_{1 \bullet}(0)\right)^{t}\left(I_{m-1}-g^{(1)}(1)\right)^{-1}\right. \\
\left.\bar{g}_{\bullet}(1+y)+\left(\bar{P}_{1 \bullet}\right)^{t}\left(I_{m-1}-P^{(1)}\right)^{-1}\left(\bar{P}_{\bullet 1}-\bar{G}_{\bullet 1}(y)\right)\right],
\end{gathered}
$$

and

$$
\begin{gathered}
\bar{\psi}_{1 \cdot}(0, y)=\frac{1}{f(0)}\left[\bar{P}_{1} \cdot-\bar{G}_{1} \cdot(y)-\left(\left(I_{m-1}-g^{(1)}(1)\right)^{-1} g^{(1)}(1+y)\right)^{t}\right. \\
\left.\bar{g}_{1} \cdot(0)+\left(\left(I_{m-1}-P^{(1)}\right)^{-1}\left(P^{(1)}-G^{(1)}(y)\right)\right)^{t} \bar{P}_{1} \cdot\right]
\end{gathered}
$$

which appears as (equation (45), (46)).

Finally $\mathcal{F}_{i}(u, x), i \in\{2, \ldots, m\}$, can be calculated from

$$
\mathcal{F}_{i}(u, x)=\sum_{v=0}^{u} \mathbb{K}(u-v) \mathcal{F}_{1}(v, x)+\mathcal{F}_{j}^{*}(u, x)
$$

Following Theorem 7 of Panjer and Wang (1993), this is a stable recursive algorithm. Next we show how to calculate explicitly $f_{1}(0, x, y)$.

\subsection{The joint distribution of the surplus prior to ruin and the severity of ruin}

We define

$$
\mathcal{F}_{i}(u, x, y)=\mathbb{P}\left(T<\infty, Z_{T} \geq-y, Z_{T-1}<x \mid Z_{0}=u, J_{0}=i\right),
$$

to be the joint distribution of the surplus prior to ruin and the severity of ruin. 
By conditioning with respect to the first period's result, the distribution (39) may be calculated from the following equations

$$
\begin{cases}\mathcal{F}_{i}(u, x, y)=\sum_{j \in\{1, m\}} \sum_{k=0}^{u+1} g_{i j}(k) \mathcal{F}_{j}(u+1-k, x)+\sum_{k=u+2}^{u+y+1} g_{i}(k) & \text { if } u<x \\ \mathcal{F}_{i}(u, x, y)=\sum_{j \in\{1, m\}} \sum_{k=0}^{u+1} g_{i j}(k) \mathcal{F}_{j}(u+1-k, x) & \text { if } u \geq x(i=1, \ldots, m) .\end{cases}
$$

Next, we define

$$
\mathcal{F}_{i}^{*}(u, x, y)=\mathbb{P}\left(T \leq \tau, Z_{T} \geq-y, Z_{T-1}<x \mid Z_{0}=u, J_{0}=i\right)(i=2, \ldots, m),
$$

to be the probability that, starting with surplus $u$ in state $i(i \in\{2, \ldots, m\})$, ruin occurs before or at the first visit to state 1 and the surplus before ruin is less than $x$ (where $x>0$ ) and the deficit is great or equal to $y$ (where $y>0$ ). Hence, by the law of total probability, we get for $u \geq 0$ and $x, y \in \mathbb{N}_{0}$

$$
\begin{cases}\mathcal{F}_{i}^{*}(u, x, y)=\sum_{j=1}^{m} \sum_{l=0}^{u+1} g_{i j}(l) \mathcal{F}_{j}^{*}(u+1-l, x)+\sum_{l=u+2}^{u+y+1} g_{i}(l) & \text { if } u<x \\ \mathcal{F}_{i}^{*}(u, x, y)=\sum_{j=1}^{m} \sum_{l=0}^{u+1} g_{i j}(l) \mathcal{F}_{j}^{*}(u+1-l, x, y) & \text { if } u \geq x .\end{cases}
$$

We define

$$
\overline{\mathcal{F}}^{*}(u, x, y)=\left(\mathcal{F}_{2}^{*}(u, x, y), \ldots, \mathcal{F}_{m}^{*}(u, x, y)\right)^{t} \in \mathbb{R}^{m-1}
$$

It is easy to show that $\left\{\mathscr{F}_{i}^{*}(u, x, y), u \in \mathbb{N}, x, y \in \mathbb{N}_{0}, i \in\{2, \ldots, m\}\right\}$ may be calculated by the following stable recursive system

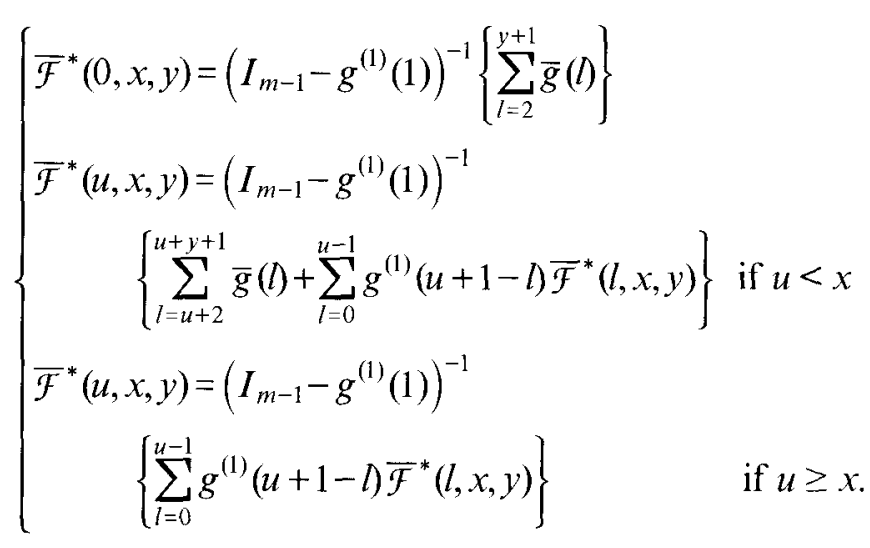


We clearly have for $u \geq 0$ and $x, y \in \mathbb{N}_{0}$ :

$$
\mathcal{F}_{i}(u, x, y)=\sum_{v=0}^{u} \mathbb{K}(u-v) \mathcal{F}_{1}(v, x, y)+\mathcal{F}_{i}^{*}(u, x, y) \quad(i \in\{2, \ldots, m\}) .
$$

By substitution of (44) into (40) with $i=1$, we obtain:

for $u<x$,

$$
\begin{aligned}
\mathcal{F}_{1}(u, x, y)= & \sum_{l=0}^{u+1} f(l) \mathcal{F}_{1}(u+1-l, x, y) \\
& +\sum_{j=2}^{m} \sum_{l=0}^{u+1} g_{1 j}(l) \mathcal{F}_{j}^{*}(u+1-l, x, y)+\sum_{v=u+2}^{u+y+1} g_{1}(v) .
\end{aligned}
$$

and, for $u \geq x$, we have

$$
\begin{aligned}
\mathcal{F}_{1}(u, x, y)= & \sum_{l=0}^{u+1} f(l) \mathcal{F}_{1}(u+1-l, x, y) \\
& +\sum_{j=2}^{m} \sum_{l=0}^{u+1} g_{1 j}(l) \mathcal{F}_{j}^{*}(u+1-l, x, y)
\end{aligned}
$$

Rearranging (45) and (46) yields for $u \in \mathbb{N}_{0}$, if $u \leq x$ :

$$
\begin{gathered}
\mathcal{F}_{1}(u, x, y)=\frac{1}{f(0)}\left[\mathcal{F}_{1}(u-1, x, y)-\sum_{l=0}^{u-1} f(u-l) \mathcal{F}_{1}(l, x, y)\right. \\
\left.-\sum_{j=2}^{m} \sum_{l=0}^{u} g_{1 j}(l) \mathcal{F}_{j}^{*}(u-l, x, y)+\sum_{v=u+1}^{u+y} g_{1}(v)\right]
\end{gathered}
$$

and if $u>x$

$$
\begin{aligned}
\mathcal{F}_{1}(u+1, x, y)= & \frac{1}{f(0)}\left[(1-f(1)) \mathcal{F}_{1}(u, x, y)-\sum_{l=0}^{u} f(u-l) \mathcal{F}_{1}(l, x, y)\right. \\
& \left.-\sum_{j=2}^{m} \sum_{l=0}^{u+1} g_{1 j}(l) \mathcal{F}_{j}^{*}(u-l, x, y)\right] .
\end{aligned}
$$

Then, following the same steps as for the proof of (32), the required explicit value for $\mathcal{F}_{1}(0, x, y)$ can be obtained by

$$
\begin{aligned}
\mathcal{F}_{1}(0, x, y) & =\frac{1}{f(0)}\left[\sum_{r=0}^{x-1} \sum_{v=r+2}^{r+y+1} g_{1}(v)-\left(\bar{g}_{1} .(0)\right)^{t}\left(I_{m-1}-g^{(1)}(1)\right)^{-1}\left\{\sum_{l=2}^{y+1} \bar{g}(l)\right\}\right. \\
+ & \left.\sum_{u=0}^{x-1} \sum_{l=u+2}^{u+y+1}\left(\bar{P}_{1}\right)^{t}\left(I_{m-1}-P^{(1)}\right)^{-1} \bar{g}(l)\right] \quad\left(x, y \in \mathbb{N}_{0}\right) .
\end{aligned}
$$


Now, the desired values for $f_{1}(0, x, y)$ are obtained by the following equations

$$
\begin{gathered}
f_{1}(0,0, y)=\frac{1}{f(0)}\left[g_{1}(y+1)-\left(\bar{g}_{1} \cdot(0)\right)^{t}\left(I_{m-1}-g^{(1)}(1)\right)^{-1} \bar{g}(y+1)\right. \\
\left.+\left(\bar{P}_{1} \cdot\right)^{t}\left(I_{m-1}-P^{(1)}\right)^{-1} \bar{g}(y+1)\right] \quad\left(y \in \mathbb{N}_{0}\right),
\end{gathered}
$$

and

$$
\begin{aligned}
& f_{1}(0, x, y) \\
& =\left(\mathcal{F}_{1}(0, x+1, y)-\mathcal{F}_{1}(0, x, y)\right)-\left(\mathcal{F}_{1}(0, x+1, y-1)-\mathcal{F}_{1}(0, x, y-1)\right) \\
& =\frac{1}{f(0)}\left[g_{1}(x+y+1)+\left(\bar{P}_{1} .\right)^{t}\left(I_{m-1}-P^{(1)}\right)^{-1} \bar{g}(x+y+1)\right]\left(x, y \in \mathbb{N}_{0}\right) .
\end{aligned}
$$

An alternative stable recursive method to calculate $\mathcal{F}_{1}(u, x, y)$ is proposed below. We use the same arguments as in the previous subsection. We can write, for $u<x$,

$$
\mathcal{F}_{1}(u, x, y)=\sum_{j=1}^{m} \sum_{k=1}^{u} \psi_{1 j}(0, k) \mathcal{F}_{j}(u-k, x, y)+\sum_{v=0}^{x-u-1} \sum_{k=u+1}^{u+y} f_{1}(0, v, k),
$$

and for $u \geq x$,

$$
\mathcal{F}_{1}(u, x, y)=\sum_{j=1}^{m} \sum_{k=1}^{u} \psi_{1 j}(0, k) \mathcal{F}_{j}(u-k, x, y)
$$

Using the formulae (52), (53) and starting from the following value

$$
\mathcal{F}_{1}(0, x, y)=\sum_{v=0}^{x-1} \sum_{k=1}^{y} f_{1}(0, v, k)
$$

allows us tó calculate $\mathcal{F}_{1}(u, x, y)$ recursively by a stable algorithm.

Finally, $\mathcal{F}_{1}(u, x, y), i \in\{2, \ldots, m\}$, can be calculated from

$$
\mathcal{F}_{i}(u, x, y)=\sum_{v=0}^{u} \mathbb{K}(u-v) \mathcal{F}_{1}(v, x, y)+\mathcal{F}_{i}^{*}(u, x, y) .
$$

We can summarize the recursive scheme to calculate $\mathcal{F}_{i}(u, x)$ for $u \in \mathbb{N}, x \in \mathbb{N}_{\ell}$ and $i \in M$, as follows:

- Step 1: Calculate the distribution $\left\{\mathbb{K}_{i}(d), d \in \mathbb{N}, i \in\{2, \ldots, m\}\right\}$ by the recursive formula (17).

- Step 2: Calculate the probabilities $\left\{\overline{\mathcal{F}}^{*}(u, k), u=0,1,2, \ldots, k=1,2, \ldots\right\}$ by the recursive formula (19). 
- Step 3: for $u=0,1,2, \ldots$, calculate

- the probabilities $\mathcal{F}_{1}(u, k)$ by the recursive formulae (33) and (35) initialized by (32). Using the values obtained for $f_{1}(0, x, y)$ by formulae $(50)$ and $(51)$, the values obtained for $\psi_{1 l}(0, v)$ with $l \in\{1, \ldots, m\}, 1 \leq v \leq u$ by formulae (36) and (37) and also the previous values obtained for $\mathcal{F}_{1}(v, k)$ and $\mathcal{F}_{i}(v, k), i \in\{2, \ldots, m\}$, with $0 \leq v \leq u-1$.

- the probabilities $\mathcal{F}_{i}(u, k), i \in\{2, \ldots, m\}$, by formula (38) using the values obtained for $\mathcal{F}_{1}(0, k), \ldots, \mathcal{F}_{1}(u, k)$.

\subsection{Application: the two-state model}

In this subsection we consider the results obtained in subsections 3.1 and 3.2 , in the case $m=2$, i.e. the environment process has only two states: state 1 and state 2 . Therefore, equations (13) become

$$
\begin{cases}\mathcal{F}_{i}(u, x)=\sum_{j \in\{1,2\}} \sum_{k=0}^{u+1} g_{i j}(k) \mathcal{F}_{j}(u+1-k, x)+\sum_{k=u+2}^{\infty} g_{i}(k) & \text { if } u<x \\ \mathcal{F}_{i}(u, x)=\sum_{j \in\{1,2\}} \sum_{k=0}^{u+1} g_{i j}(k) \mathcal{F}_{j}(u+1-k, x) & \text { if } u \geq x(i=1,2) .\end{cases}
$$

It follows from (17) that $\left\{\mathbb{\mathbb { S } _ { 2 }}(d), d \in \mathbb{N}\right\}$ may be calculated by the following stable recursive system

$$
\left\{\begin{array}{l}
\mathbb{K}_{2}(0)=\frac{g_{21}(1)}{1-g_{22}(1)} \\
\mathbb{K}_{2}(d)=\frac{1}{1-g_{22}(1)}\left\{g_{21}(d+1)+\sum_{l=0}^{d-1} g_{22}(d+1-l) \mathbb{K}_{2}(l)\right\} \quad(d=1,2, \ldots) .
\end{array}\right.
$$

and from (19) we get the following recursive stable system for $\mathcal{F}_{2}^{*}(u, k)$ :

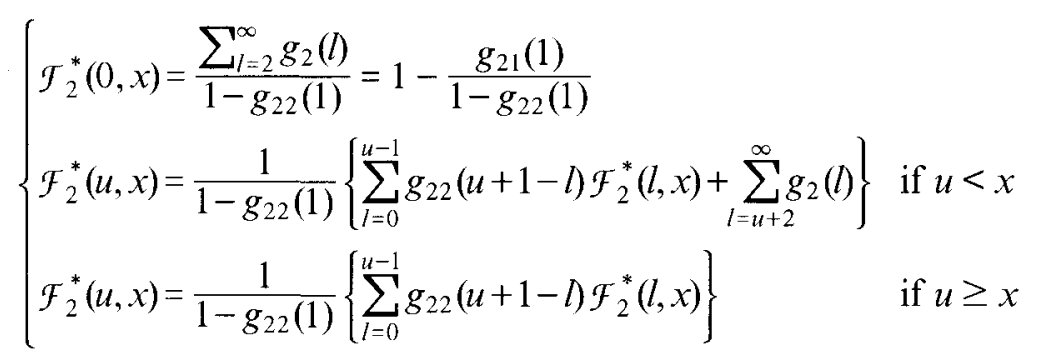

Equation (20) is here equivalent to

$$
\mathcal{F}_{2}(u, x)=\sum_{v=0}^{u} \mathbb{K}_{2}(u-v) \mathcal{F}_{1}(v, x)+\mathcal{F}_{2}^{*}(u, x)
$$


Then, the required stable recursive system is based on the following formula, for $u<x$

$$
\mathcal{F}_{1}(u, x)=\sum_{j=1}^{2} \sum_{y=1}^{u} \psi_{1 j}(0, y) \mathcal{F}_{j}(u-y, x)+\sum_{v=0}^{x-u-1} \sum_{y=u+1}^{\infty} f_{1}(0, v, y),
$$

and, for $u \geq x$

$$
\mathcal{F}_{1}(u, x)=\sum_{j=1}^{2} \sum_{y=1}^{u} \psi_{1 j}(0, y) \mathcal{F}_{j}(u-y, x)
$$

To start the recursive scheme (60) and (61), we use formula (32) which is here equivalent to

$$
\begin{aligned}
\mathcal{F}_{1}(0, x)=\frac{1}{f(0)}\left[\sum_{l=0}^{x-1}\left(1-G_{1}(l+1)\right)-g_{12}(0) \frac{\sum_{v=2}^{\infty} g_{2}(v)}{1-g_{22}(1)}\right. \\
\left.+\frac{p_{12}}{p_{21}}\left(\sum_{r=0}^{x-1}\left(1-G_{2}(l+1)\right)\right)\right],
\end{aligned}
$$

where

$$
f(0)=g_{11}(0)+g_{12}(0) \mathbb{K}_{2}(0) .
$$

The joint density of the surplus prior to ruin and the severity of ruin with no initial surplus is given by

$$
f_{1}(0,0, y)=\frac{1}{f(0)}\left[g_{1}(y+1)-g_{12}(0) \frac{g_{2}(u+1)}{1-g_{22}(1)}+\frac{p_{12}}{p_{21}} g_{2}(y+1)\right] \quad\left(\forall y \in \mathbb{N}_{0}\right),
$$

and

$$
f_{1}(0, x, y)=\frac{1}{f(0)}\left[g_{1}(x+y+1)+\frac{p_{12}}{p_{21}} g_{2}(x+y+1)\right] \quad\left(\forall x, y \in \mathbb{N}_{0}\right) .
$$

Example 1. We will use the same example as in Reinhard and Snoussi (2000). Let $m=2, c=1$ and the distribution of the claim given by

\begin{tabular}{ccccc}
\hline \hline $\boldsymbol{k}$ & $\boldsymbol{g}_{\mathbf{1 1}}(\boldsymbol{k})$ & $\boldsymbol{g}_{\mathbf{1 2}}(\boldsymbol{k})$ & $\boldsymbol{g}_{\mathbf{2 1}}(\boldsymbol{k})$ & $\boldsymbol{g}_{\mathbf{2 2}}(\boldsymbol{k})$ \\
\hline 0 & $5 / 8$ & 0 & 0 & 0 \\
1 & $1 / 8$ & $1 / 8$ & 0 & $1 / 6$ \\
2 & $1 / 8$ & 0 & $1 / 2$ & $1 / 6$ \\
3 & 0 & 0 & $1 / 6$ & 0 \\
$\geq 4$ & 0 & 0 & 0 & 0 \\
\hline \hline
\end{tabular}


We easily can find:

$$
\gamma^{*}=\frac{14}{19}
$$

Reinhard and Snoussi (2000) give an explicit value for the survival probabilities as follows:

$$
\Phi_{1}(u)=1-\frac{1}{2}\left(\frac{3}{5}\right)^{u} \quad(u \in \mathbb{N})
$$

and

$$
\left\{\begin{array}{l}
\Phi_{2}(0)=0 \\
\Phi_{2}(u)=1-\frac{7}{10}\left(\frac{3}{5}\right)^{u-1} \quad\left(u \in \mathbb{N}_{0}\right)
\end{array}\right.
$$

\begin{tabular}{|c|c|c|c|c|}
\hline \multicolumn{5}{|c|}{ state 1} \\
\hline $\boldsymbol{u}$ & $F_{1}(u, 1)$ & $\mathcal{F}_{1}(u, 2)$ & $\psi_{1}(u)$ & Exact value of $\psi_{1}(u)$ \\
\hline 0 & 0.45 & 0.5 & 0.5 & 0.5 \\
\hline 1 & 0.23 & 0.3 & 0.3 & 0.3 \\
\hline 2 & 0.138 & 0.18 & 0.18 & 0.18 \\
\hline 3 & 0.0828 & 0.108 & 0.108 & 0.108 \\
\hline 4 & 0.04968 & 0.0648 & 0.0648 & 0.0648 \\
\hline 5 & 0.029808 & 0.03888 & 0.03888 & 0.03888 \\
\hline 6 & 0.0178848 & 0.023328 & 0.023328 & 0.023328 \\
\hline 7 & 0.01073088 & 0.0139968 & 0.0139968 & 0.0139968 \\
\hline 8 & 0.006438528 & 0.00839808 & 0.00839808 & 0.00839808 \\
\hline 9 & 0.003863117 & 0.005038848 & 0.005038848 & 0.005038848 \\
\hline 10 & 0.00231787 & 0.003023309 & 0.003023309 & 0.003023309 \\
\hline 20 & 0.000014015 & 0.000018281 & 0.000018281 & 0.000018281 \\
\hline 30 & 0.000000085 & 0.000000111 & 0.000000111 & 0.000000111 \\
\hline \multicolumn{5}{|c|}{ state 2} \\
\hline $\boldsymbol{u}$ & $\mathcal{F}_{2}(u, 1)$ & $\mathcal{F}_{2}(u, 2)$ & $\psi_{2}(u)$ & Exact value of $\psi_{2}(u)$ \\
\hline 0 & 1 & 1 & 1 & 1 \\
\hline 1 & 0.47 & 0.7 & 0.7 & 0.7 \\
\hline 2 & 0.322 & 0.42 & 0.42 & 0.42 \\
\hline 3 & 0.1932 & 0.252 & 0.252 & 0.252 \\
\hline 4 & 0.11592 & 0.1512 & 0.1512 & 0.1512 \\
\hline 5 & 0.069552 & 0.09072 & 0.09072 & 0.09072 \\
\hline 6 & 0.0417312 & 0.054432 & 0.054432 & 0.054432 \\
\hline 7 & 0.02503872 & 0.0326592 & 0.0326592 & 0.0326592 \\
\hline 8 & 0.015023232 & 0.01959552 & 0.01959552 & 0.01959552 \\
\hline 9 & 0.009013939 & 0.0011757312 & 0.0011757312 & 0.0011757312 \\
\hline 10 & 0.005408364 & 0.007054387 & 0.007054387 & 0.007054387 \\
\hline 20 & 0.000032702 & 0.000042655 & 0.000042655 & 0.000042655 \\
\hline 30 & 0.000000198 & 0.000000258 & 0.000000258 & 0.000000258 \\
\hline
\end{tabular}

Table 1 shows some values obtained through formulae (60), (61) and (62).

TABLE 1. $\left(\gamma^{*}=0.7368\right)$ 
The last column in Table 1 presents the exact probabilities of ruin obtained by (63) and (64). It may be checked that the algorithm produced the same figures as those produced by (63) and (64).

Example 2. We consider the case when $m=2, c=1$ and the individual claim amount distribution is given by

\begin{tabular}{cllllll}
\hline \hline $\boldsymbol{k}$ & \multicolumn{1}{c}{$\boldsymbol{0}$} & \multicolumn{1}{c}{$\boldsymbol{1}$} & \multicolumn{1}{c}{$\boldsymbol{2}$} & \multicolumn{1}{c}{$\boldsymbol{3}$} & \multicolumn{1}{c}{$\boldsymbol{4}$} & \multicolumn{1}{c}{$\mathbf{5}$} \\
\hline$g_{11}(k)$ & 0,725 & 0,025 & 0,025 & 0 & 0,025 & 0 \\
$g_{12}(k)$ & 0,15 & 0,025 & 0 & 0 & 0 & 0,025 \\
$g_{21}(k)$ & 0 & 0,75 & 0 & 0,025 & 0,025 & 0 \\
$g_{22}(k)$ & 0 & 0,15 & 0,025 & 0,025 & 0 & 0 \\
\hline
\end{tabular}

The numerical illustrations of this example are presented in Table 2.

TABLE 2. $\left(\gamma^{*}=0.5\right)$

\begin{tabular}{|c|c|c|c|c|c|c|}
\hline state & $u$ & $\mathcal{F}_{i}(u, 1)$ & $\mathcal{F}_{i}(u, 2)$ & $\mathcal{F}_{i}(u, 3)$ & $\mathcal{F}_{i}(u, 4)$ & $\psi_{i}(u)$ \\
\hline$i=1$ & 0 & 0,096054889 & 0,176243568 & 0,241852487 & 0,271012007 & 0,271012 \\
\hline$i=2$ & & 0,202401372 & 0,273156089 & 0,331046312 & 0,356775300 & 0,356775 \\
\hline$i=1$ & 1 & 0,014813660 & 0,088141276 & 0,160767192 & 0,193045377 & 0,193045 \\
\hline$i=2$ & & 0,019023858 & 0,174041010 & 0,239825355 & 0,269062841 & 0,269063 \\
\hline$i=1$ & 2 & 0,011857080 & 0,028494864 & 0,101135424 & 0,135707139 & 0,135707 \\
\hline$i=2$ & & 0,019799780 & 0,043479017 & 0,142552555 & 0,175531303 & 0,175531 \\
\hline$i=1$ & 3 & 0,011704526 & 0,026087196 & 0,043810219 & 0,080586749 & 0,080587 \\
\hline$i=2$ & & 0,014730234 & 0,037191787 & 0,061744240 & 0,097831000 & 0,097831 \\
\hline$i=1$ & 4 & 0,009419522 & 0,021597044 & 0,035621150 & 0,044674645 & 0.044675 \\
\hline$i=2$ & & 0,010111365 & 0,024859361 & 0,045142174 & 0,057128063 & 0,057128 \\
\hline$i=1$ & 5 & 0,003492980 & 0,011939612 & 0,022278398 & 0,028846359 & 0,028846 \\
\hline$i=2$ & & 0,004505665 & 0,013965340 & 0,027064235 & 0,036371874 & 0,036372 \\
\hline$i=1$ & 6 & 0,002481850 & 0,006189992 & 0,012785043 & 0,017512815 & 0,017513 \\
\hline$i=2$ & & 0,003241076 & 0,008006138 & 0,015740855 & 0,021886641 & 0,021887 \\
\hline$i=1$ & 7 & 0,001601092 & 0,004143631 & 0,007478344 & 0,010290206 & 0,01029 \\
\hline$i=2$ & & 0,002020353 & 0,005288737 & 0,009560440 & 0,012955462 & 0,012955 \\
\hline$i=1$ & 8 & 0,000927499 & 0,002501553 & 0,004703196 & 0,006240559 & 0,006241 \\
\hline$i=2$ & & 0,001148860 & 0,003131502 & 0,005925312 & 0,007894649 & 0,007895 \\
\hline$i=1$ & 9 & 0,000540570 & 0,001463136 & 0,002807821 & 0,003809303 & 0,003809 \\
\hline$i=2$ & & 0,000690272 & 0,001842587 & 0,003528934 & 0,004792124 & 0,004792 \\
\hline$i=1$ & 10 & 0,000341347 & 0,000889726 & 0,001679750 & 0,002288714 & 0,002289 \\
\hline$i=2$ & & 0,000429651 & 0,001126796 & 0,002118479 & 0,002878793 & 0,002879 \\
\hline$i=1$ & 15 & 0,000027071 & 0,000072069 & 0,000136002 & 0,000183783 & 0,000184 \\
\hline$i=2$ & & 0,000034076 & 0,000090703 & 0,000171269 & 0,000231503 & 0,000232 \\
\hline$i=1$ & 20 & 0,000002176 & 0,000005782 & 0,000010917 & 0,000014768 & 0,000015 \\
\hline$i=2$ & & 0,000002741 & 0,000007283 & 0,000013749 & 0,000018598 & 0,000019 \\
\hline
\end{tabular}

The value of $\psi_{i}(u)$ is obtained by the stable recursive method presented by Reinhard and Snoussi (2000). 


\section{REFERENCES}

Asmussen, S. (1989). Risk theory in a markovian environment. Scand. Actuarit J., 66-100.

Bowers, N.L., Gerber, H.V., Hickman, J.C., Jones, D.A. and NesbitT, C.J. (1987). Actuarial Mathematics. Society of Actuaries, Itasca, Illinois.

DiCKSON, D.C.M. (1992). On the distribution of the surplus prior to ruin. Insurance: Mathematics and Economics 11, 191-207.

DiCKSON, D.C.M. and WATERS, H.R. (1992). The probability and severity of ruin in finite and infinite time. Astin Bulletin 23, 177-190.

Dickson, D.C.M., Egidio Dos Reis, A.D. and Waters, H.R. (1995). Some stable algorithms in ruin theory and their applications. Astin Bulletin 25, 153-175.

Dufresne, F. (1989). Probabilité et sévérité de la ruine modèle classique de la théorie du risque collectif et une de ses extensions. Ph.D. thesis, Université de Lausanne.

Dufresne, F. and Gerber, H.U. (1988). The surplus immediately before and at ruin, and the amount of the claim causing ruin. Insurance: Mathematics and Economics 7, 193-199.

Ger ber, H.U. (1979). An Introduction to Mathematical Risk Theory. Monograph No. 8, S.S. Huebner Foundation, Distributed by R. Irwin, Homewood, IL.

Gerber, H.U. (1988). Mathematical fun with the compound binomial process. Astin Bulletin 18, $161-168$.

Gerber, H.U. and Shiu, E.S.W. (1997). The joint distribution of the time of ruin, the surplus immediately before ruin, and the deficit at ruin. Insurance: Mathematics and Economics 21, 129-137.

Gerber, H.U. and Shiu, E.S.W. (1998). On the time value of ruin. North American Actuarial Journal $2,48.78$

Grandell, J. (1991). Aspects of Risk Theory. Springer Series in Statistics.

JANSSEN, J. (1970). Sur une généralisation du concept de promenade aléatoire sur la droite réelle. Ann. Ins. H. Poincaré, B VI, 249-269.

Janssen, J. and Reinhard, J.M. (1985). Probabilités de ruine pour une classe de modèles de risque semi-Markoviens. Astin Bulletin 15, 123-133.

Klugman, A.S., Panjer, H.H. and Wilmot, G.E. (1998). Loss Models: From Data to Decisions. Wiley Series in Probability and Statistics.

Newbould, M. (1973). A classification of a random walk defined on a finite Markov chain. Z. Wahrscheinlichkeitstheorie verw. Geb. 26, 95-104.

PANJER, H.H. and WANG, S. (1993). On the stability of recursive formulas. Astin Bulletin 23, 227-258.

Reinhard, J.M. (1984). On a class of semi-Markov risk models obtained as classical risk models in a Markovian environment. Astin Bulletin 14, 23-43.

Reinhard, J.M. and SNoussi, M. (1998). The severity of ruin in a discrete semi Markov risk model. Technical Report 94, ISRO, Université Libre de Bruxelles.

REINHARD, J.M. and SNoussi, M. (2000). The probability of ruin in a discrete semi Markov risk model. To appear in Blätter Deutsche Gesellschaft für Versicherungsmathematik.

SNoussi, M. (1998). The severity of ruin in the Markov-Modulated risk models. Proceedings of the 2nd International Symposium on Semi-Markov Models: Theory and Applications. Ed. by J. Janssen and N. Limnios, Compiegne, 377-382.

WILLMOT, G.E. and LiN, X.S. (1998). Exact and approximate properties of the distribution of surplus before and after ruin. Insurance: Mathematics and Economics 23, 91-110.

\section{Mohammed SNoussi}

Secura s.a.

Montoyer, 12

B-1000 Bruxelles

Belgique
JEAN MARIE REINHARD

Université Libre de Bruxelles

Campus de la plaine CP 210

B-1050 Bruxelles

Belgique 